\title{
Asymptotic Expansions of Multiple Integrals of Rapidly Oscillating Functions
}

\author{
By T. Iwaniec and A. Lutoborski
}

Abstract. Expansions of multiple integrals

$$
\int_{a_{1}}^{b_{1}} \ldots \int_{a_{n}}^{b_{n}} w\left(\sigma_{1} x_{1}, \ldots, \sigma_{n} x_{n}\right) g\left(x_{1}, \ldots, x_{n}\right) d x_{1} \cdots d x_{n},
$$

where $w$ is a function on $\mathbf{R}^{n}$ which is $\left(b_{k}-a_{k}\right)$-periodic in the $k$ th variable, $k=$ $1, \ldots, n$, and $g$ is smooth, are given in terms of negative powers of the integers $\sigma_{1}, \ldots, \sigma_{n}$. Estimates of the remainder term in the expansion are also given.

Introduction. Let $w$ be a function with integrable $p$ th power and $g$ a smooth function on an interval $Q$ in $\mathbf{R}^{n}$. Denote by $\bar{w}$ the periodic extension of $w$ to $\mathbf{R}^{n}$. Set $\sigma x=\left(\sigma_{1} x_{1}, \ldots, \sigma_{n} x_{n}\right)$ for $x \in \mathbf{R}^{n}$ and $\sigma=\left(\sigma_{1}, \ldots, \sigma_{n}\right)$, an $n$-tuple of integers. Note that if $\sigma_{1}, \ldots, \sigma_{n}$ are large then $x \mapsto \bar{w}(\sigma x)$ is a rapidly oscillating function.

Our purpose is to obtain an expansion of the integral

$$
I_{\sigma}(w ; g)=\int_{Q} \bar{w}(\sigma x) g(x) d x
$$

in terms of negative powers of $\sigma_{1}, \ldots, \sigma_{n}$. We recall the standard result, cf. [3], characterizing the asymptotic behavior of $I_{\sigma}(w ; g)$ as

$$
\begin{aligned}
& \lim _{\sigma_{1} \rightarrow+\infty} \int_{Q} \bar{w}(\sigma x) g(x) d x=\left(\frac{1}{|Q|} \int_{Q} w(x) d x\right)\left(\int_{Q} g(x) d x\right) . \\
& \vdots \\
& \sigma_{n} \rightarrow+\infty
\end{aligned}
$$

The right-hand side of this equality is exactly the zero-order term in our expansion. We find explicitly the higher-order terms which involve the integrals of the derivatives of $g$ and the coefficients dependent on the moments of $w$. This is why we say that our expansion is of Euler-Maclaurin type. We also derive estimates of the magnitude of the remainder in the expansion and give sufficient conditions for convergence of the infinite expansion. Finally, we note that the expansion formulas for the multiple integral $I_{\sigma}(w ; g)$ are essentially based on the study of the boundary value problem

$$
\operatorname{div} Z=f
$$

where $f \in L^{p}(Q)$ is given and $Z=\left[Z_{1}, \ldots, Z_{n}\right]$ is an unknown vector field whose $k$ th component has zero trace on the $k$ th face of $Q$. We solve explicitly this problem, which seems to be of independent interest.

Received February 18, 1987.

1980 Mathematics Subject Classification (1985 Revision). Primary 41A60; Secondary 35C15. 
1. Preliminaries and Euler-Maclaurin Type Expansions of Integrals. For ease of reference we begin by listing some standard function spaces used in this article. Let $\Omega$ be a nonempty, open subset of $\mathbf{R}^{n}$. We denote by $L^{p}(\Omega), 1 \leq p \leq \infty$, the usual space of real-valued functions $f$ satisfying $\|f\|_{p}=\left(\int_{\Omega}|f(x)|^{p} d x\right)^{1 / p}<\infty$ if $1 \leq p<\infty$, and if $p=\infty$, then $L^{\infty}(\Omega)$ denotes the space of essentially bounded functions with norm $\|f\|_{\infty}$.

The Sobolev space $W^{m, p}(\Omega), 0 \leq m$ and $1 \leq p \leq \infty$, consists of functions $f$ satisfying $\|f\|_{m, p}=\left(\int_{\Omega} \sum_{|\gamma|=0}^{m}\left|D^{\gamma} f(x)\right|^{p} d x\right)^{1 / p}<\infty$ for $1 \leq p<\infty$ and $\|f\|_{m, \infty}=$ $\max _{0 \leq|\gamma| \leq m}\left\|D^{\gamma} f\right\|_{\infty}<\infty$, where $D^{\gamma}=D_{1}^{\gamma_{1}} \cdots D_{n}^{\gamma_{n}}$ for $\gamma=\left(\gamma_{1}, \ldots, \gamma_{n}\right)$ and $D_{k}$ denotes the partial derivative with respect to $x_{k}$. As usual, $W_{0}^{m, p}(\Omega)$ denotes the closure of $C_{0}^{\infty}(\Omega)$ in $W^{m, p}(\Omega)$.

We begin with a brief summary of results concerning Euler-Maclaurin type expansions of a class of integrals of functions defined on the interval $Q=(0,1)$ and introduced in [2]. We define linear operators $P: L^{p}(Q) \rightarrow L^{p}(Q), T: L^{p}(Q) \rightarrow$ $L^{p}(Q), S: L^{p}(Q) \rightarrow W^{1, p}(Q)$ and $Z: L^{p}(Q) \rightarrow W_{0}^{1, p}(Q)$ by the formulas:

$$
\begin{aligned}
P f & =\int_{0}^{1} f(t) d t \\
(T f)(x) & =f(x)-\int_{0}^{1} f(t) d t \\
(S f)(x) & =\int_{0}^{x} f(t) d t \\
(Z f)(x) & =\int_{0}^{x}\left[\int_{0}^{1} f(t) d t-f(s)\right] d s,
\end{aligned}
$$

for every $f \in L^{p}(Q)$. We note that $T=\mathrm{Id}-P$, where Id is the identity operator, $Z=-S T$ and $P T=0$. If $w \in L^{p}(Q)$ then $(Z w)(0)=(Z w)(1)=0$ and $(Z w)^{\prime}=$ $P w-w$, so that indeed $Z w \in W_{0}^{1, p}(Q)$. Hence, for any $g \in W^{1, q}(Q)$, where $q$ satisfies $1 / p+1 / q=1$, integration by parts yields

$$
\int_{0}^{1} w(x) g(x) d x-\int_{0}^{1} w(x) d x \int_{0}^{1} g(x) d x=\int_{0}^{1}(Z w)(x) g^{\prime}(x) d x .
$$

The above expansion formula can be used recursively in computing the righthand side integral in (1.5). To do this, set $Z^{0}=\mathrm{Id}, Z^{1}=Z$ and $Z^{j}=Z Z^{j-1}$ for $j \geq 1$ and $a_{j}(w)=\int_{0}^{1}\left(Z^{j} w\right)(x) d x$, then

$$
\int_{0}^{1} w(x) g(x) d x=\sum_{j=0}^{N} a_{j}(w) \int_{0}^{1} g^{(j)}(x) d x+\int_{0}^{1} Z^{N+1}(w)(x) g^{(N+1)}(x) d x
$$

for any $g \in W^{N+1, q}(Q)$.

Expansion (1.6) of the integral $\int_{0}^{1} w(x) g(x)$ is referred to as an Euler-Maclaurin type expansion. In [2] the coefficients $a_{j}(w)$ were expressed in terms of moments of $w$, an estimate of $\left\|Z_{N+1}\right\|_{\infty}$ was obtained and the relation between (1.6) and Euler-Maclaurin quadratures, cf. [4], was investigated. However, a more detailed analysis of the norms of the operators $T$ and $Z$ is necessary before expansions of multidimensional integrals can be studied. 
LEMMA 1. If $p \in[1, \infty]$ then

$$
\begin{gathered}
\|T\|_{p} \leq 2^{|1-(2 / p)|} \leq 2 \\
\|Z\|_{p} \leq \frac{1}{\pi}\left(\frac{\pi}{2}\right)^{|1-(2 / p)|} \leq \frac{1}{2},
\end{gathered}
$$

and the first inequality in both (1.7) and (1.8) is sharp for $p=1,2$ and $p=\infty$.

Proof. We first prove (1.7). We begin with $p=1,2$. Let $f \in L^{1}(Q)$; then

$$
\|T f\|_{1} \leq\|f\|_{1}+\left|\int_{0}^{1} f(t) d t\right| \leq 2\|f\|_{1} .
$$

This estimate is sharp. Indeed if $\chi_{(0, \varepsilon]}$ denotes the characteristic function of the interval $(0, \varepsilon]$, then for $f=\chi_{(0, \varepsilon]}$, where $0<\varepsilon<1$, we have that $T f=$ $(1-\varepsilon) \chi_{(0, \varepsilon]}-\varepsilon \chi_{(\varepsilon, 1)}$ and hence $\|T f\|_{1}=2(1-\varepsilon)\|f\|_{1}$. In the case $p=2$, for $f \in L^{2}(Q)$ we have

$$
\|T f\|_{2}^{2}=\|f\|_{2}^{2}-\left|\int_{0}^{1} f(t) d t\right|^{2} \leq\|f\|_{2}^{2},
$$

and equality holds only if $f$ has zero average. In the case $f \in L^{\infty}(Q)$ it is clear that

$$
\|T f\|_{\infty} \leq\|f\|_{\infty}+\left|\int_{0}^{1} f(t) d t\right| \leq 2\|f\|_{\infty},
$$

and the estimate is sharp because for $f=\chi_{(0, \varepsilon]}-\chi_{(\varepsilon, 1)}$ we have $T f=2(1-\varepsilon) \chi_{(0, \varepsilon]}-$ $2 \varepsilon \chi_{(\varepsilon, 1)}$ and hence $\|T f\|_{\infty}=2(1-\varepsilon)=2(1-\varepsilon)\|f\|_{\infty}$. In the case of intermediate $p \notin\{1,2, \infty\}$, inequality (1.7) is obtained by an interpolation argument based on the M. Riesz-Thorin theorem.

We next prove (1.8). The proof for $p=2$ is based on the following sharp Sobolev inequality, cf. [7],

$$
\|u\|_{2} \leq \frac{1}{\pi}\left\|u^{\prime}\right\|_{2}, \quad \forall u \in W_{0}^{1,2}(Q) .
$$

Setting $u=Z f$, where $f \in L^{2}(Q)$, in that inequality, and using (1.10), we obtain

$$
\|Z f\|_{2} \leq \frac{1}{\pi}\left\|(Z f)^{\prime}\right\|_{2}=\frac{1}{\pi}\|T f\|_{2} \leq \frac{1}{\pi}\|f\|_{2} .
$$

Equality will hold for $f(x)=\cos \pi x$ since

$$
Z f(x)=-\frac{1}{\pi} \sin \pi x \text { and }\|Z f\|_{2}=\frac{1}{\pi}\|\sin \pi x\|_{2}=\frac{1}{\pi}\|\cos \pi x\|_{2}=\frac{1}{\pi}\|f\|_{2} .
$$

To prove (1.8) when $p=1$ and $p=\infty$, we write

$$
(Z f)(x)=\int_{0}^{1} K(x, t) f(t) d t
$$

where

$$
K(x, t)=\left\{\begin{array}{cl}
1-x & \text { if } 0<t \leq x \\
-x & \text { if } x<t<1 .
\end{array}\right.
$$

We note that $\int_{0}^{1}|K(x, t)| d t=2 x(1-x) \leq \frac{1}{2}$ and also $\int_{0}^{1}|K(x, t)| d x=t^{2}-t+\frac{1}{2} \leq \frac{1}{2}$.

Now if $f \in L^{\infty}(Q)$ we obtain that

$$
|(Z f)(x)| \leq\|f\|_{\infty} \int_{0}^{1}|K(x, t)| d t \leq \frac{1}{2}\|f\|_{\infty}
$$


or equivalently that

$$
\|Z f\|_{\infty} \leq \frac{1}{2}\|f\|_{\infty}
$$

Equality will hold in (1.14) if $f=\chi_{(0,1 / 2]}-\chi_{(1 / 2,1)}$ because $(Z f)(x)=-x_{(0,1 / 2]}-$ $(1-x) \chi_{(1 / 2,1)}$ and $\|Z f\|_{\infty}=\frac{1}{2}=\frac{1}{2}\|f\|_{\infty}$. If $f \in L^{1}(Q)$ we have

$$
\|Z f\|_{1} \leq \int_{0}^{1}\left[|f(t)| \int_{0}^{1}|K(x, t)| d x\right] d t \leq \frac{1}{2}\|f\|_{1} .
$$

We can see that (1.15) is sharp by taking $f=\chi_{(0, \varepsilon]}-\chi_{(1-\varepsilon, 1)}$ for $0<\varepsilon \leq \frac{1}{2}$, for which $(Z f)(x)=-x \chi_{(0, \varepsilon]}-\varepsilon \chi_{[\varepsilon, 1-\varepsilon]}-(1-x) \chi_{(1-\varepsilon, 1)}$. Thus, $\|Z f\|_{1}=\varepsilon-\varepsilon^{2}=$ $\left(\frac{1}{2}-\frac{\varepsilon}{2}\right)\|f\|_{1}$.

Finally, (1.8) for the intermediate case $p \notin\{1,2, \infty\}$ follows by an interpolation argument. Namely, if $2<p<\infty$, then for $\alpha=2 / p$ the interpolation inequality is

$$
\|Z\|_{p} \leq\|Z\|_{2}^{\alpha}\|Z\|_{\infty}^{1-\alpha}=\left(\frac{1}{\pi}\right)^{2 / p}\left(\frac{1}{2}\right)^{1-2 / p}=\frac{1}{\pi}\left(\frac{\pi}{2}\right)^{|1-2 / p|} .
$$

Similarly, if $1<p<2$, then for $\beta=2-2 / p$,

$$
\|Z\|_{p} \leq\|Z\|_{2}^{\beta}\|Z\|_{1}^{1-\beta}=\left(\frac{1}{\pi}\right)^{2-2 / p}\left(\frac{1}{2}\right)^{2 / p-1}=\frac{1}{\pi}\left(\frac{\pi}{2}\right)^{|1-2 / p|} .
$$

Remark. For $p \notin\{1,2, \infty\}$ we do not know the norms $\|T\|_{p}$ and $\|Z\|_{p}$. We believe, though, that the method used in [7] for a related problem could be applied.

Remark. If $\mathcal{L}: L^{p}(a, b) \rightarrow W_{0}^{1, p}(a, b), 1 \leq p \leq \infty$, is defined by

$$
\mathcal{L} f(x)=\int_{a}^{x}\left[\frac{1}{b-a} \int_{a}^{b} f(t) d t-f(s)\right] d s,
$$

then, using (1.8), we obtain that

$$
\|\mathcal{L}\|_{p} \leq \frac{1}{2}(b-a) .
$$

2. A Decomposition of a Function with Zero Average into Functions with Zero Partial Averages. Let $\mathcal{N}=\{1,2, \ldots, n\}$; we then denote by $I=$ $\left\{i_{1}, \ldots, i_{k}\right\}, k \leq n$, a subset of $\mathcal{N}$ containing $k$ elements, for which we write $|I|=k$.

We use lower-case Greek letters for multi-indices, e.g., $\gamma=\left(\gamma_{1}, \ldots, \gamma_{n}\right)$, where $\gamma_{i}$ are nonnegative integers and $|\gamma|=\gamma_{1}+\cdots+\gamma_{n}$. The standard notation is used: $\gamma \leq$ $\alpha$ when $\gamma_{i} \leq \alpha_{i}$ for each $1 \leq i \leq n ; \gamma !=\gamma_{1} ! \ldots \gamma_{n}$ ! denotes the multidimensional factorial and $\left(\begin{array}{l}\alpha \\ \gamma\end{array}\right)=\left(\begin{array}{l}\alpha_{1} \\ \gamma_{1}\end{array}\right) \ldots\left(\begin{array}{l}\alpha_{n} \\ \gamma_{n}\end{array}\right)$ denotes the multidimensional binomial coefficient. As usual, $e_{k}$ is the standard "basis" multi-index $e_{k}=(0, \ldots, 1, \ldots, 0)$.

Let $Q$ be an interval in $\mathbf{R}^{n}, n \geq 1$, i.e.,

$$
Q=\left\{x=\left(x_{1}, \ldots, x_{n}\right): a_{i}<x_{i}<b_{i}, i \in \mathcal{N}\right\},
$$

where $a_{i}, b_{i}$ are finite, $a_{i}<b_{i}$ for $i \in \mathcal{N}$, and let $\bar{Q}$ denote the closure of $Q$.

We will write $x^{\gamma}=x_{1}^{\gamma_{1}} \cdots x_{n}^{\gamma_{n}}$ and $\sigma x=\left(\sigma_{1} x_{1}, \ldots, \sigma_{n} x_{n}\right)$ for any multi-indices $\gamma$ and $\sigma$.

The sets $Q_{k}^{+}=\left\{x \in \bar{Q}: x_{k}=b_{k}\right\}$ and $Q_{k}^{-}=\left\{x \in \bar{Q}: x_{k}=a_{k}\right\}$ for $1 \leq k \leq n$ are called faces of $Q$, and these sets constitute the boundary $\partial Q=\bigcup_{k=1}^{n}\left(Q_{k}^{+} \cup Q_{k}^{-}\right)$ of $Q$. 
For $i \in \mathcal{N}, L_{i}^{p}(Q)$ stands for the subspace of $L^{p}(Q)$ consisting of functions independent of the $x_{i}$-variable. More generally, $L_{I}^{p}(Q)=L_{i_{1}}^{p}(Q) \cap \cdots \cap L_{i_{k}}^{p}(Q)$ if $I=$ $\left\{i_{1}, \ldots, i_{k}\right\}$. Thus $L_{I}^{p}(Q)$ contains those functions in $L^{p}(Q)$ which are independent of $x_{i_{1}}, \ldots, x_{i_{k}}$.

We introduce now the operators $P_{i}: L^{p}(Q) \rightarrow L_{i}^{p}(Q)$ for $i \in \mathcal{N}$ by

$$
\left(P_{i} f\right)(x)=\frac{1}{b_{i}-a_{i}} \int_{a_{i}}^{b_{i}} f(x) d x_{i}
$$

and call them averaging projectors. For $I=\left\{i_{1}, \ldots, i_{k}\right\}$ we denote $P_{I}: L^{p}(Q) \rightarrow$ $L_{I}^{p}(Q)$, where

$$
P_{I}=P_{i_{1}} P_{i_{2}} \cdots P_{i_{k}} \text {. }
$$

Consequently, $P_{\mathcal{N}} f=|Q|^{-1} \int_{Q} f(x) d x$, and we set $P_{\varnothing}=$ Id. The mean value $|Q|^{-1} \int_{Q} f(x) d x$ will also be written as $f_{Q} f(x) d x$. Here $|Q|$ denotes the volume of $Q$, that is, $|Q|=\prod_{i=1}^{n}\left(b_{i}-a_{i}\right)$.

It follows from Fubini's theorem that the operators $P_{I}$ and $P_{J}$ commute and that

$$
P_{I} P_{J}=P_{I \cup J}
$$

for all $I \subset \mathcal{N}$ and $J \subset \mathcal{N}$.

Moreover, for every $f \in L^{P}(Q)$ and $g \in L^{q}(Q)$, where $1 \leq p, q \leq \infty$ and $1 / p+1 / q=1$, we have

$$
\int_{Q} f(x)\left(P_{I} g\right)(x) d x=\int_{Q}\left(P_{I} f\right)(x) g(x) d x .
$$

To see this, denote $d x_{I}=d x_{i_{1}} \cdots d x_{i_{k}}$ for $I=\left\{i_{1}, \ldots, i_{k}\right\}$ and

$$
\begin{aligned}
\int_{Q} f\left(P_{I} g\right) d x & =\int\left[\int f\left(P_{I} g\right) d x_{I}\right] d x_{\mathcal{N}-I}=\int\left[\left(P_{I} g\right) \int f d x_{I}\right] d x_{\mathcal{N}-I} \\
& =\int\left[\left(\int g d x_{I}\right)\left(P_{I} f\right)\right] d x_{\mathcal{N}-I}=\int\left[\int\left(P_{I} f\right) g d x_{I}\right] d x_{\mathcal{N}-I} \\
& =\int_{Q}\left(P_{I} f\right) g d x .
\end{aligned}
$$

If $p=2$ then $P_{I}$ is the orthogonal projector from $L^{2}(Q)$ onto $L_{I}^{2}(Q)$, while Id $-P_{I}$ is the projector onto ker $P_{I}$. Moreover, by (2.4), $P_{I}$ is selfadjoint. We find that

$$
\left\|P_{I}\right\|_{p}=1
$$

for $I \subset \mathcal{N}$ and $1 \leq p \leq \infty$. Consequently,

$$
\left\|\mathrm{Id}-P_{I}\right\|_{p} \leq 2 \text {. }
$$

The last inequality is sharp only for $p=1$ and $p=\infty$ and $I \neq \varnothing$. Indeed, if $p=2$ we have $\left\|\mathrm{Id}-P_{I}\right\|_{2}=1$. Hence by interpolation,

$$
\left\|\mathrm{Id}-P_{I}\right\|_{p} \leq 2^{|1-2 / p|} \leq 2
$$

for all $1 \leq p \leq \infty$.

Next we introduce the operators $T_{k}$, which play a key role further on. For $1 \leq k \leq n$, we set

$$
T_{k}=\left(\mathrm{Id}-P_{k}\right) T
$$


where

$$
T=\frac{1}{n} \sum_{s=0}^{n-1}\left(\begin{array}{c}
n-1 \\
s
\end{array}\right)^{-1} \sum_{|I|=s} P_{I}
$$

Note that

$$
\left(\mathrm{Id}-P_{k}\right) P_{I}= \begin{cases}P_{I}-P_{I \cup\{k\}} & \text { if } k \notin I, \\ 0 & \text { if } k \in I .\end{cases}
$$

Therefore, $T_{k}$ can be written as

$$
T_{k}=\frac{1}{n} \sum_{s=0}^{n-1}\left(\begin{array}{c}
n-1 \\
s
\end{array}\right)^{-1} \sum_{\substack{|I|=s \\
k \notin I}}\left(P_{I}-P_{I \cup\{k\}}\right) .
$$

We state now the basic properties of operators $T_{k}$.

PROPOSITION 1. The following identities hold:

$$
\sum_{k=1}^{n} T_{k}=\mathrm{Id}-P_{\mathcal{N}}
$$

and, for all $1 \leq k \leq n$,

$$
P_{k} T_{k}=0 .
$$

Proof. Assertion (2.11) follows directly from the definition (2.7). Next, by simple combinatorial arguments, we see that

$$
\sum_{k=1}^{n} \sum_{\substack{|I|=s \\ k \notin I}} P_{I \cup\{k\}}=(s+1) \sum_{|J|=s+1} P_{J}
$$

and

$$
\sum_{k=1}^{n} \sum_{\substack{|I|=s \\ k \notin I}} P_{I}=(n-s) \sum_{|J|=s} P_{J}
$$

for $0 \leq s \leq n-1$.

To prove (2.10), we change indices in the summation in (2.9):

$$
\begin{aligned}
\sum_{k=1}^{n} T_{k}= & \frac{1}{n} \sum_{s=0}^{n-1}\left(\begin{array}{c}
n-1 \\
s
\end{array}\right)^{-1}\left[(n-s) \sum_{|J|=s} P_{J}-(s+1) \sum_{|J|=s+1} P_{J}\right] \\
= & \frac{1}{n} \sum_{s=0}^{n-1}(n-s)\left(\begin{array}{c}
n-1 \\
s
\end{array}\right)^{-1} \sum_{|J|=s} P_{J}-\left(\frac{1}{n}\right) \sum_{s=1}^{n} s\left(\begin{array}{c}
n-1 \\
s-1
\end{array}\right)^{-1} \sum_{|J|=s} P_{J} \\
= & \sum_{|J|=0} P_{J}-\sum_{|J|=n} P_{J} \\
& \quad+\frac{1}{n} \sum_{s=1}^{n-1}\left[(n-s)\left(\begin{array}{c}
n-1 \\
s
\end{array}\right)^{-1}-s\left(\begin{array}{c}
n-1 \\
s-1 ;
\end{array}\right] \sum_{|J|=s} P_{J}\right. \\
= & \mathrm{Id}-P_{\mathcal{N}}
\end{aligned}
$$

since $(n-s)\left(\begin{array}{c}n-1 \\ s\end{array}\right)^{-1}=s\left(\begin{array}{c}n-1 \\ s-1\end{array}\right)^{-1}$ for $1 \leq s \leq n-1$ in the last summation. 
The norms of the operators $T_{k}$ can be estimated using (2.5) and (2.6). Clearly,

$$
T_{k}=\left(\mathrm{Id}-P_{k}\right) \frac{1}{n} \sum_{s=0}^{n-1}\left(\begin{array}{c}
n-1 \\
s
\end{array}\right)^{-1} \sum_{\substack{|I|=s \\
k \notin I}} P_{I}
$$

and hence

$$
\begin{aligned}
\left\|T_{k}\right\|_{p} & \leq\left\|\mathrm{Id}-P_{k}\right\|_{p} \frac{1}{n} \sum_{s=0}^{n-1}\left(\begin{array}{c}
n-1 \\
s
\end{array}\right)^{-1} \sum_{\substack{|I|=s \\
k \notin I}} 1 \\
& =\left\|\mathrm{Id}-P_{k}\right\|_{p} \leq 2^{|1-2 / p|},
\end{aligned}
$$

for $1 \leq k \leq n$ and every $1 \leq p \leq \infty$.

The operators $T_{k}$ provide us with an explicit decomposition of a function with zero average into functions with zero partial averages:

LEMma 2. (A Decomposition Lemma.) For every $w \in L^{p}(Q)$, where $1 \leq p \leq$ $\infty$, and for $1 \leq k \leq n$,

$$
\begin{gathered}
\sum_{k=1}^{n} T_{k} w=w-f_{Q} w(x) d x \\
P_{k} T_{k} w=0 \\
\left\|T_{k} w\right\|_{p} \leq 2^{|1-2 / p|}\|w\|_{p} .
\end{gathered}
$$

Furthermore, the decomposition (2.14) is optimal in $L^{2}(Q)$, that is, if $w \in L^{2}(Q)$ and $w_{k} \in L^{2}(Q)$ are such that

$$
\sum_{k=1}^{n} w_{k}=w-f_{Q} w(x) d x
$$

and

$$
P_{k} w_{k}=0 \quad \text { for } 1 \leq k \leq n,
$$

then

$$
\sum_{k=1}^{n}\left\|T_{k} w\right\|_{2}^{2} \leq \sum_{k=1}^{n}\left\|w_{k}\right\|_{2}^{2}
$$

Proof. The relations (2.14) and (2.15) follow automatically from (2.10) and (2.11) in Proposition 1, while (2.16) follows from (2.13). To prove optimality (2.19), we denote $v_{k}=T_{k} w-w_{k}$. Clearly, $\sum_{k=1}^{n} v_{k}=0$ and $P_{k} v_{k}=0$ for $1 \leq k \leq n$. Then, since $T_{k}$ are selfadjoint,

$$
\begin{aligned}
\int_{Q} \sum_{k=1}^{n} v_{k} T_{k} w & =\int_{Q} w \sum_{k=1}^{n} T_{k} v_{k}=\int_{Q} w \sum_{k=1}^{n} T\left(\mathrm{Id}-P_{k}\right) v_{k} \\
& =\int_{Q} w \sum_{k=1}^{n} T v_{k}=\int_{Q} w T\left(\sum_{k=1}^{n} v_{k}\right)=0
\end{aligned}
$$

This shows that

$$
\int_{Q} \sum_{k=1}^{n}\left|T_{k} w\right|^{2}=\int_{Q} \sum_{k=1}^{n} w_{k} T_{k} w
$$


Using the Schwarz inequality, we find that

$$
\int_{Q} \sum_{k=1}^{n}\left|T_{k} w\right|^{2} \leq\left(\int_{Q} \sum_{k=1}^{n}\left|T_{k} w\right|^{2}\right)^{1 / 2}\left(\int_{Q} \sum_{k=1}^{n}\left|w_{k}\right|^{2}\right)^{1 / 2}
$$

which completes the proof of (2.19).

3. Expansion of Euler-Maclaurin Type. The averaging projectors will now be used to solve the following problem: Given $f \in L^{p}(Q)$, find a vector field $Z=\left[Z_{1}, \ldots, Z_{n}\right], Z_{i} \in L^{p}(Q), 1 \leq i \leq n$, such that

$$
\int_{Q} \sum_{k=1}^{n} Z_{k}(x) D_{k} g(x) d x=-\int_{Q} f(x) g(x) d x,
$$

for every $g \in W^{1, q}(Q), 1 \leq p, q \leq \infty, 1 / p+1 / q=1$.

Note that the test functions $g$ are not required to be in $W_{0}^{1, q}(Q)$. That is why the necessary condition of solvability of $(3.1)$ is $\int_{Q} f(x) d x=0$. Clearly, (3.1) implies the equation

$$
\operatorname{div} Z=\sum_{k=1}^{n} D_{k} Z_{k}=f
$$

where the derivatives $D_{k} Z_{k}$ are understood in the sense of distributions.

In what follows, for $f=f_{Q} w(x) d x-w$ we construct explicitly a solution $Z=$ $\left[Z_{1}, \ldots, Z_{n}\right]$ of $(3.2)$ such that $Z_{k}, 1 \leq k \leq n$, has partial derivative $D_{k} Z_{k}$ in $L^{p}(Q)$ and zero trace on $Q_{k}^{+} \cup Q_{k}^{-}$. For such a solution $Z$, the equations (3.1) and (3.2) will be equivalent.

We define first the partial antiderivative operators $S_{k}$,

$$
\left(S_{k} f\right)(x)=\int_{a_{k}}^{x_{k}} f\left(x_{1}, \ldots, x_{k-1}, t, x_{k+1}, \ldots, x_{n}\right) d t
$$

for $x=\left(x_{1}, \ldots, x_{n}\right) \in Q, 1 \leq k \leq n$.

Directly from the definition, we have

$$
\left(S_{k} f\right)(x)= \begin{cases}P_{k} f(x) & \text { if } x \in Q_{k}^{+} \\ 0 & \text { if } x \in Q_{k}^{-}\end{cases}
$$

For $w \in L^{p}(Q)$ we introduce the vector field $Z(w), Z(w)=\left[Z_{1} w, \ldots, Z_{n} w\right]$, which is defined on $Q$ by

$$
Z_{k} w=-S_{k} T_{k} w \text { for } 1 \leq k \leq n
$$

or more explicitly,

$$
Z_{k} w=\frac{1}{n} \sum_{s=0}^{n-1}\left(\begin{array}{c}
n-1 \\
s
\end{array}\right)^{-1} \sum_{|I|=s} S_{k}\left(P_{I \cup\{k\}}-P_{I}\right) w .
$$

Lemma 2 implies that

$$
\operatorname{div} Z(w)=-\sum_{k=1}^{n} T_{k} w=f_{Q} w(x) d x-w
$$


and therefore $Z(w)$ defined in (3.5) solves (3.2) for $f=f_{Q} w(x) d x-w$. From (3.4) and (2.15), $Z_{k}(w)$ vanishes in $Q_{k}^{+} \cup Q_{k}^{-}$, so integrating by parts for any $g \in W^{1, q}(Q)$, we get

$$
-\int_{Q} \operatorname{div} Z(w)(x) g(x) d x=\int_{Q} \sum_{k=1}^{n}\left(Z_{k} w\right)(x) D_{k} g(x) d x
$$

Hence we obtained the first-order terms in the expansion of Euler-Maclaurin type:

LEMMA 3. If $w \in L^{p}(Q)$ and if $g \in W^{1, q}(Q), 1 \leq p, q \leq \infty, 1 / p+1 / q=1$, then

$$
\int_{Q} w(x) g(x) d x-f_{Q} w(x) d x \int_{Q} g(x) d x=\sum_{k=1}^{n} \int_{Q}\left(Z_{k} w\right)(x) D_{k} g(x) d x
$$

We can now analyze the recursive use of the formula (3.9).

LEMMA 4. (Higher-Order Expansion of Euler-Maclaurin Type.) If $w \in L^{p}(Q)$ and $g \in W^{N+1, q}(Q), 1 \leq p, q \leq \infty, 1 / p+1 / q=1$, then

$$
\begin{aligned}
\int_{Q} w(x) g(x) d x= & \sum_{0 \leq|\gamma| \leq N} a_{\gamma}(w) \int_{Q} D^{\gamma} g(x) d x \\
& +\sum_{|\gamma|=N+1} \int_{Q}\left(Z_{\gamma} w\right)(x) D^{\gamma} g(x) d x,
\end{aligned}
$$

where the operators $Z_{\gamma}: L^{p}(Q) \rightarrow L^{p}(Q)$ are defined by

$$
Z_{\gamma}= \begin{cases}\text { Id } & \text { if } \gamma=(0, \ldots, 0), \\ Z_{k} & \text { if } \gamma=(0, \ldots, 1, \ldots, 0)=e_{k}, \\ \sum_{k=1}^{n} Z_{k} Z_{\gamma-e_{k}} & \text { if } \gamma \geq(0, \ldots, 0),\end{cases}
$$

and $Z_{\gamma}$ is null if at least one component of the multi-index $\gamma$ is negative. The coefficients $a_{\gamma}(w)$ for $\gamma \geq(0, \ldots, 0)$ are given by

$$
a_{\gamma}(w)=f_{Q}\left(Z_{\gamma} w\right)(x) d x
$$

and can be computed explicitly in terms of moments of $w$ by

$$
a_{\gamma}(w)=\frac{1}{\gamma !} \sum_{\rho \leq \gamma}\left(\begin{array}{l}
\gamma \\
\rho
\end{array}\right) \mathbf{B}_{\gamma-\rho}(b-a)^{\gamma-\rho} f_{Q}(x-a)^{\rho} w(x) d x,
$$

where $\mathbf{B}_{\alpha}=B_{\alpha_{1}} \cdots B_{\alpha_{n}}$ and $B_{k}$ is the kth Bernoulli number.

Proof. We prove (3.10) by induction with respect to $N$. When $N=0$ then (3.10) reduces to (3.9). Suppose that (3.10) holds for $N-1$, that is,

$$
\int_{Q} w(x) g(x) d x=\sum_{0 \leq|\gamma| \leq N-1} a_{\gamma}(w) \int_{Q} D^{\gamma} g(x) d x+\sum_{|\gamma|=N} \int_{Q}\left(Z_{\gamma} w\right)(x) D^{\gamma} g(x) d x \text {. }
$$

We now apply Lemma 3:

$$
\begin{aligned}
\int_{Q}\left(Z_{\gamma} w\right)(x) D^{\gamma} g(x) d x= & f_{Q}\left(Z_{\gamma} w\right)(x) d x \int_{Q} D^{\gamma} g(x) d x \\
& +\sum_{k=1}^{n} \int_{Q}\left(Z_{k} Z_{\gamma} w\right)(x) D_{k} D^{\gamma} g(x) d x .
\end{aligned}
$$


Summing up the above equalities over all $|\gamma|=N$, we get

$$
\begin{aligned}
\sum_{|\gamma|=N} \int_{Q}\left(Z_{\gamma} w\right)(x) D^{\gamma} g(x) d x= & \sum_{|\gamma|=N} a_{\gamma}(w) \int_{Q} D^{\gamma} g(x) d x \\
& +\sum_{|\mu|=N+1} \int_{Q} \sum_{k=1}^{n}\left(Z_{k} Z_{\mu-e_{k}} w\right)(x) D^{\mu} g(x) d x \\
= & \sum_{|\gamma|=N} a_{\gamma}(w) \int_{Q} D^{\gamma} g(x) d x \\
& +\sum_{|\mu|=N+1} \int_{Q}\left(Z_{\mu} w\right)(x) D^{\mu} g(x) d x .
\end{aligned}
$$

This proves (3.10) and (3.12). Finally, (3.13) is obtained by testing (3.10) with $g=g_{\gamma}$ a product of scaled Bernoulli polynomials, cf. [1], namely we set

$$
g_{\gamma}(x)=\frac{1}{|Q|} \sum_{\rho \leq \gamma}\left(\begin{array}{l}
\gamma \\
\rho
\end{array}\right) \mathbf{B}_{\gamma-\rho}(x-a)^{\rho}(b-a)^{\gamma-\rho} .
$$

Substituting $g_{\gamma}$ into (3.10) we obtain (3.13), since we can check that

$$
\int_{Q} D^{\beta} g_{\gamma}(x) d x= \begin{cases}0 & \text { if }(0, \ldots, 0) \leq \beta \leq \gamma, \gamma \neq \beta \\ \gamma ! & \text { if } \beta=\gamma .\end{cases}
$$

Remark. (3.11) yields that for any $s \geq 0, Z_{\gamma}=\sum_{|\alpha|=s} Z_{\alpha} Z_{\gamma-\alpha}$.

It is also possible to obtain the estimate of the norm of the operator $Z_{\gamma}$ which generalizes the one-dimensional estimate (1.16).

PROPOSITION 2. If $1 \leq p \leq \infty$ then

$$
\left\|Z_{\gamma}\right\|_{p} \leq \frac{|\gamma| !}{2^{|\gamma|} \gamma !}(b-a)^{\gamma}
$$

for any multi-index $\gamma$.

Proof. Induction with respect to $|\gamma|$ : If $|\gamma|=0$ then $Z_{\gamma}=$ Id and (3.14) is an equality. If $|\gamma|=1$, say $\gamma=e_{k}$, then $Z_{\gamma}=Z_{k}$, and then by (1.16) $\left\|Z_{k}\right\|_{p} \leq$ $\frac{1}{2}\left(b_{k}-a_{k}\right)$. For $|\gamma|>1, \gamma=\left(\gamma_{1}, \ldots, \gamma_{n}\right)$, we obtain

$$
\begin{aligned}
\left\|Z_{\gamma}\right\|_{p} & =\left\|\sum_{k=1}^{n} Z_{k} Z_{\gamma-e_{k}}\right\|_{p} \leq \sum_{k=1}^{n}\left\|Z_{k}\right\|_{p}\left\|Z_{\gamma-e_{k}}\right\|_{p} \\
& \leq \sum_{k=1}^{n} \frac{1}{2}\left(b_{k}-a_{k}\right) \frac{(|\gamma|-1) ! \gamma_{k}}{2^{|\gamma|-1} \gamma !} \frac{(b-a)^{\gamma}}{b_{k}-a_{k}}=\frac{|\gamma| !}{2^{|\gamma|} \gamma !}(b-a)^{\gamma} .
\end{aligned}
$$

The question of the validity of expansion (3.10) as $N \rightarrow \infty$ is another problem. A set of sufficient conditions for convergence are given in

PROPOSITION 3. Let $1 \leq p, q \leq \infty, 1 / p+1 / q=1$; then for every $w \in L^{p}(Q)$,

$$
\int_{Q} w(x) g(x) d x=\sum_{0 \leq|\gamma|} a_{\gamma}(w) \int_{Q} D^{\gamma} g(x) d x
$$


provided $g \in C^{\infty}(Q)$ satisfies the following growth condition:

$$
\left\|D^{\gamma} g\right\|_{q} \leq c \frac{d^{\gamma}}{(b-a)^{\gamma}},
$$

for any $\gamma=\left(\gamma_{1}, \ldots, \gamma_{n}\right)$, where $c>0$ and $d=\left(d_{1}, \ldots, d_{n}\right), d_{j}>0, d_{1}+\cdots+d_{n}<2$.

Proof. Naturally, the main step in proving the convergence of $(3.10)$ is to estimate the remainder term $R_{N+1}(w ; g)$, where

$$
R_{N+1}(w ; g)=\sum_{|\gamma|=N+1} \int_{Q}\left(Z_{\gamma} w\right)(x) D^{\gamma} g(x) d x .
$$

Using (3.14) and the binomial formula, we obtain

$$
\begin{aligned}
\left|R_{N+1}(w ; g)\right| & \leq \sum_{|\gamma|=N+1}\left\|Z_{\gamma} w\right\|_{p}\left\|D^{\gamma} g\right\|_{q} \\
& \leq \sum_{|\gamma|=N+1}\left(\frac{|\gamma| !}{2^{|\gamma|} \gamma !}(b-a)^{\gamma} \frac{c d^{\gamma}}{(b-a)^{\gamma}}\right)\|w\|_{p} \\
& =c\|w\|_{p}\left(\frac{d_{1}+\cdots+d_{n}}{2}\right)^{N+1},
\end{aligned}
$$

and hence, because of the condition $d_{1}+\cdots+d_{n}<2$, it follows that

$$
\lim _{N \rightarrow \infty} R_{N+1}(w ; g)=0 \text {. }
$$

4. Asymptotic Expansion of Integrals of Rapidly Oscillating Functions. The expansion in powers of $m^{-1}$ of the Fourier integrals

$$
\int_{0}^{1} \cos (2 \pi m x) g(x) d x, \quad \int_{0}^{1} \sin (2 \pi m x) g(x) d x
$$

of a smooth function $g$ are well known, cf. [6], [5]. If $m$ is large, the integrands are usually referred to as rapidly oscillating functions and, for example,

$$
\begin{aligned}
\int_{0}^{1} \cos (2 \pi m x) g(x) d x= & \sum_{q=1}^{p-1} \frac{(-1)^{q-1}}{(2 \pi m)^{2 q}} \int_{0}^{1} g^{(2 q)}(x) d x \\
& +\frac{(-1)^{p}}{(2 \pi m)^{2 p}} \int_{0}^{1}(\cos (2 \pi m x)-1) g^{(2 p)}(x) d x
\end{aligned}
$$

Expansions of integrals $\int_{0}^{1} w(m x) g(x) d x$ with more general rapidly oscillating factors $w(m x)$ of period $m^{-1}$ were derived and studied in [2]. Our purpose is to derive similar asymptotic expansions for multiple integrals of rapidly oscillating functions on an interval in $\mathbf{R}^{n}$. Let $Q=\left\{x=\left(x_{1}, \ldots, x_{n}\right): a_{i}<x_{i}<b_{i}, i \in \mathcal{N}\right\}$ be an interval and $h: Q \rightarrow \mathbf{R}$. By $\bar{h}$ we mean the periodic extension of $h$, which is defined almost everywhere on $\mathbf{R}^{n}$, that is $\bar{h}(x)=h(x)$ for $x \in Q$ and $\bar{h}(x)=\bar{h}\left(x+\left(b_{k}-a_{k}\right) e_{k}\right)$ for $1 \leq k \leq n$ and almost every $x \in \mathbf{R}^{n}$. For our purposes, there is no need to define $\bar{h}$ on the whole $\mathbf{R}^{n}$. This is because $\bar{h}$ is used under an integral sign.

We will consider an integral $I_{\sigma}(w ; g)=\int_{Q} \bar{w}(\sigma x) g(x) d x$, where $w \in L^{p}(Q), \sigma x=$ $\left(\sigma_{1} x_{1}, \ldots, \sigma_{n} x_{n}\right)$ and $\sigma=\left(\sigma_{1}, \ldots, \sigma_{n}\right)$ is a multi-index with big components. Such integrals occur as energy integrals in mechanics of composite media, $\mathrm{cf}$. [3]. 
Our expansion formula of Euler-Maclaurin type is given in

THEOREM 1. Let $w \in L^{p}(Q)$ and $g \in W^{N+1, q}(Q)$, where $1 / p+1 / q=1,1 \leq$ $p, q \leq \infty$. Then

$$
\begin{aligned}
\int_{Q} \bar{w}(\sigma x) g(x) d x= & \sum_{0 \leq|\gamma| \leq N} \frac{1}{\sigma^{\gamma}} a_{\gamma}(w) \int_{Q} D^{\gamma} g(x) d x \\
& +\int_{Q} \sum_{|\gamma|=N+1} \frac{1}{\sigma^{\gamma}}\left(\overline{Z_{\gamma} w}\right)(\sigma x) D^{\gamma} g(x) d x,
\end{aligned}
$$

where $a_{\gamma}(w), Z_{\gamma} w$ are defined in (3.12) and (3.11), and $\sigma=\left(\sigma_{1}, \ldots, \sigma_{n}\right)$ is an arbitrary multi-index.

Proof. Expansion (4.1) follows from Lemma 4 by standard scaling arguments. Namely, applying (3.10) to the function $W(x)=\bar{w}(\sigma x)$, one gets

$$
\begin{aligned}
\int_{Q} \bar{w}(\sigma x) g(x) d x= & \sum_{0 \leq|\gamma| \leq N} a_{\gamma}(W) \int_{Q} D^{\gamma} g(x) d x \\
& +\int_{Q} \sum_{|\gamma|=N+1} \frac{1}{\sigma^{\gamma}}\left(Z_{\gamma} W\right)(x) D^{\gamma} g(x) d x .
\end{aligned}
$$

What remains to be proved is that for $x \in Q$

$$
\left(Z_{\gamma} W\right)(x)=\frac{1}{\sigma^{\gamma}}\left(\overline{Z_{\gamma} w}\right)(\sigma x) .
$$

According to the recursive definition of $Z_{\gamma}$ it is sufficient to show that

for each $k=1, \ldots, n$.

$$
\left(Z_{k} W\right)(x)=\frac{1}{\sigma_{k}}\left(\overline{Z_{k} w}\right)(\sigma x)
$$

The above, however, follows from $\left(T_{k} W\right)(x)=\overline{T_{k} w}(\sigma x)$ and $Z_{k}=-S_{k} T_{k}$ by an affine change of variable. Finally,

$$
\begin{aligned}
a_{\gamma}(W) & =f_{Q}\left(Z_{\gamma} W\right)(x) d x=\frac{1}{\sigma^{\gamma}} f_{Q}\left(\overline{Z_{\gamma} w}\right)(\sigma x) d x \\
& =\frac{1}{\sigma^{\gamma}} f_{Q}\left(Z_{\gamma} w\right)(x) d x=\frac{1}{\sigma^{\gamma}} a_{\gamma}(w) .
\end{aligned}
$$

We note that the standard result on the asymptotic behavior of $I_{\sigma}(w ; g)$, cf. [3, page 9] is

$$
\begin{aligned}
& \lim _{\sigma_{1} \rightarrow \infty} \int_{Q} \bar{w}(\sigma x) g(x) d x=f_{Q} w(x) d x \int_{Q} g(x) d x . \\
& \vdots \\
& \sigma_{n} \rightarrow \infty
\end{aligned}
$$

This limit agrees with the zero-order term in (4.1). From Theorem 1 there follows directly the following:

PROPOSITION 4. Let $w \in L^{\infty}(Q)$, and let $g \in W^{N+1,1}\left(\mathbf{R}^{n}\right)$; then

$$
\begin{aligned}
\int_{\mathbf{R}^{n}} \bar{w}(\sigma x) g(x) d x= & \sum_{0 \leq|\gamma| \leq N} \frac{1}{\sigma^{\gamma}} a_{\gamma}(w) \int_{\mathbf{R}^{n}} D^{\gamma} g(x) d x \\
& +\int_{\mathbf{R}^{n}} \sum_{|\gamma|=N+1} \frac{1}{\sigma^{\gamma}}\left(\overline{Z_{\gamma} w}\right)(\sigma x) D^{\gamma} g(x) d x
\end{aligned}
$$

for any multi-index $\sigma=\left(\sigma_{1}, \ldots, \sigma_{n}\right)$. 
It is to be noted that (4.4) remains true also when $\sigma_{1}, \ldots, \sigma_{n}$ are arbitrary positive numbers not necessarily integers. If $N=0,(4.4)$ gives that

$$
\begin{aligned}
& \lim _{\sigma_{1} \rightarrow \infty} \int_{\mathbf{R}^{n}} \bar{w}(\sigma x) g(x) d x=\left(f_{Q} w(x) d x\right)\left(\int_{\mathbf{R}^{n}} g(x) d x\right), \\
& \vdots \\
& \sigma_{n} \rightarrow \infty
\end{aligned}
$$

which for $n=1$ is known as Féjer's formula.

It is also possible to give a set of sufficient conditions for the convergence of the expansion in Theorem 1.

Proposition 5. Let $1 \leq p, q \leq \infty, 1 / p+1 / q=1, w \in L^{p}(Q)$, and assume that $g \in C^{\infty}(Q)$ satisfies the growth condition: There exists a vector $c=\left(c_{1}, \ldots, c_{n}\right)$ such that $\left\|D^{\gamma} g\right\|_{q} \leq c^{\gamma}$ for any $\gamma=\left(\gamma_{1}, \ldots, \gamma_{n}\right)$. Then

$$
\int_{Q} \bar{w}(\sigma x) g(x) d x=\sum_{0 \leq|\gamma|} \frac{1}{\sigma^{\gamma}} a_{\gamma}(w) \int_{Q} D^{\gamma} g(x) d x
$$

provided $\sigma_{1}, \ldots, \sigma_{n}$ are large enough to satisfy

$$
\sum_{k=1}^{n} \frac{c_{k}\left(b_{k}-a_{k}\right)}{2 \sigma_{k}}<1
$$

Proof. Along the lines of Proposition 3, denote

$$
R_{N+1}(w ; g)=\int_{Q} \sum_{|\gamma|=N+1} \frac{1}{\sigma^{\gamma}}\left(\overline{Z_{\gamma} w}\right)(\sigma x) D^{\gamma} g(x) d x
$$

since $\int_{Q}\left|\left(\overline{Z_{\gamma} w}\right)(\sigma x)\right|^{p} d x=\int_{Q}\left|Z_{\gamma} w(x)\right|^{p} d x$ we have that

$$
\begin{aligned}
\left|R_{N+1}(w ; g)\right| & \leq \sum_{|\gamma|=N+1} \frac{1}{\sigma^{\gamma}}\left(\int_{Q}\left|\left(\overline{Z_{\gamma} w}\right)(\sigma x)\right|^{p} d x\right)^{1 / p}\left\|D^{\gamma} g\right\|_{q} \\
& \leq\left(\sum_{|\gamma|=N+1} \frac{|\gamma| !(b-a)^{\gamma}}{\sigma^{\gamma} \cdot 2^{|\gamma|} \gamma !} c^{\gamma}\right)\|w\|_{p} \\
& =\left(\sum_{k=1}^{n} \frac{c_{k}\left(b_{k}-a_{k}\right)}{2 b_{k}}\right)^{N+1}\|w\|_{p}
\end{aligned}
$$

An interesting discussion of the pitfalls in using asymptotic expansions of the type (4.6) directly in numerical integration is given in [5]. Troubles arise when the assumptions of Proposition 5 are violated.

Department of Mathematics

Syracuse University

Syracuse, New York 13244-1150

1. M. Abramowitz \& I. A. Stegun, Handbook of Mathematical Functions, Dover, New York, 1972.

2. U. BANERJEe, L. J. LARDY \& A. LUtOBORSKI, "Asymptotic expansions of integrals of certain rapidly oscillating functions," Math. Comp., v. 49, 1987, pp. 243-249. 
3. A. Bensoussan, J. L. Lions \& G. Papanicolaou, Asymptotic Analysis of Periodic Structures, North-Holland, Amsterdam, 1978.

4. P. J. DAVIS \& P. RABINOWitz, Methods of Numerical Integration, 2nd ed., Academic Press, New York, 1984.

5. J. N. LYNESS, "The calculation of Fourier coefficients by the Möbius inversion of the Poisson summation formula, Part I. Functions whose early derivatives are continuous," Math. Comp., v. 24, 1970, pp. 101-135.

6. H. J. STETTER, "Numerical approximation of Fourier transforms," Numer. Math., v. 8, 1966, pp. 235-249.

7. G. Talenti, "Best constant in Sobolev inequality," Ann. Mat. Pura Appl., v. 110, 1976, pp. 353-372. 